\title{
Alkaline phosphatase in pediatric patients with genu varum caused by vitamin D-deficient rickets
}

\author{
Masashi Mukai ${ }^{1)}$, Takehisa Yamamoto ${ }^{1)}$, Shinji Takeyari²), Yasuhisa Ohata ${ }^{2)}$, Taichi Kitaoka ${ }^{2)}$, \\ Takuo Kubota $^{2)}$, Katsusuke Yamamoto ${ }^{3)}$, Eri Kijima ${ }^{1)}$, Yasuhiro Hasegawa ${ }^{1)}$, Toshimi Michigami ${ }^{4)}$ and \\ Keiichi Ozono ${ }^{2)}$ \\ 1) Department of Pediatrics, Minoh City Hospital, Osaka, Japan \\ 2) Department of Pediatrics, Osaka University Graduate School of Medicine, Osaka, Japan \\ 3) Department of Pediatric Nephrology and Metabolism, Osaka Women's and Children's Hospital, Osaka, Japan \\ 4) Department of Bone and Mineral Research, Osaka Women's and Children's Hospital, Osaka, Japan
}

\begin{abstract}
An elevated serum alkaline phosphatase (ALP) level is one of the markers for the presence of rickets in children, but it is also associated with bone formation. However, its role in diagnosing genu varum in pediatric patients with vitamin Ddeficient rickets is still unknown. To clarify the role of the serum ALP level in assessing the severity of genu varum, we retrospectively investigated this issue statistically using data on rickets such as serum intact parathyroid hormone (iPTH), 25hydroxyvitamin $\mathrm{D}, 1,25$-dihydroxyvitamin $\mathrm{D}$, ALP, the level of creatinine as the percentage of the median according to age $(\% \mathrm{Cr})$, and the metaphyseal diaphyseal angle (MDA) in the lower extremities as an index of the severity of genu varum. A multiple regression analysis revealed that $\log \mathrm{ALP}$ and $\% \mathrm{Cr}$ values were negatively associated with MDA values. The former association was also confirmed by a linear mixed model, while iPTH was positively associated with MDA by path model analysis. To elucidate the association of ALP with MDA in the presence of $\mathrm{PPTH}$, we investigated three-dimensional figures by neural network analysis. This indicated the presence of a biphasic association of ALP with MDA: the first phase increases while the second decreases MDA. The latter phenomenon is considered to be associated with the increase in bone formation due to the mechanical stress loaded on the lower extremities. These findings are important and informative for pediatricians to understand the significance of the serum ALP level in pediatric patients with genu varum caused by vitamin D deficiency.
\end{abstract}

Key words: Genu varum, Alkaline phosphatase, Linear mixed model analysis, Structural equation modeling analysis, Neural network analysis

GENU VARUM is classified into physiological and pathological cases [1]. The causes of the latter consist of calcium and/or vitamin D deficiency followed by Blount's disease, metabolic bone diseases, and achondroplasia [1]. The increase in the metaphyseal diaphyseal angle (MDA: angle between the transverse line of the metaphysis of the proximal tibia and the perpendicular to the long axis of the tibial diaphysis) has recently been used as an index for the quantitative evaluation of genu varum [2]. However, there have been no studies on the relationships between MDA and markers of bone metabolism except for a study that showed a weak positive correlation between MDA and serum alkaline phosphatase

Submitted Oct. 6, 2020; Accepted Feb. 10, 2021 as EJ20-0622 Released online in J-STAGE as advance publication Mar. 24, 2021 Correspondence to: Takehisa Yamamoto, MD, Department of Pediatrics, Minoh City Hospital, 5-7-1 Kayano, Minoh City, Osaka 562-0014, Japan.

E-mail: t.yamamoto@minoh-hp.jp
(ALP) levels [3]. However, it included only pediatric patients with physiological genu varum. Thus, no studies to date have investigated the relationship between serum ALP levels and MDA values in patients with vitamin Ddeficient rickets.

In this study, we evaluated the degree of genu varum in pediatric patients with vitamin D-deficient rickets by MDA, and analyzed its association with serum metabolic bone markers such as intact parathyroid hormones (iPTH), the ALP level, and also serum creatinine level, a marker of muscle mass [4]. We found that an increase in the serum ALP level had an opposite association with MDA: ALP was associated with aggravating genu varum in the early stage of rickets, but its amelioration in the advanced stage of rickets based on serum iPTH levels [5]. These findings may be useful for pediatricians to understand the role of the serum ALP level in genu varum in order to determine appropriate therapy for pediatric patients with vitamin D-deficient rickets. 


\section{Materials and Methods}

We retrospectively reviewed clinical records, serum laboratory data, and roentgenograms of children who visited the Department of Pediatrics of Minoh City Hospital, Osaka Women's and Children's Hospital, and Osaka University Hospital from 1997 to 2012. We included children with vitamin D-deficient rickets aged older than 11 months in this study. The diagnostic criterion for vitamin D-deficient rickets was as follows: serum 25(OH)D level less than $20 \mathrm{ng} / \mathrm{mL}$ in the presence of bone lesions. If the serum $25(\mathrm{OH}) \mathrm{D}$ level was not measured, we clinically diagnosed vitamin D-deficient rickets when patients had bone lesions with a serum iPTH level greater than $60 \mathrm{pg} / \mathrm{mL}$ in addition to the exclusion of the following diseases: hypophosphatemic rickets, hypercalciuria, and metabolic bone diseases [6]. The serum intact PTH concentration was determined by an ECLIA method. Serum $1,25(\mathrm{OH})_{2} \mathrm{D}$ and $25(\mathrm{OH}) \mathrm{D}$ levels were measured by a radioreceptor assay and competitive protein binding assay, respectively. We excluded children who could not stand by themselves and also visited our hospitals fewer than three times. The study protocol was conducted using the opt-out method. The ethics review board of the above three hospitals approved the study and also waived the need for informed consent. All methods were performed per the Declaration of Helsinki and relevant guidelines.

The diagnosis of genu varum was confirmed by a lower limb roentgenogram. The development of lower limb deformity was evaluated by the average value of MDA on the left and right. The definition of genu varum was a minimum MDA value of 11 degrees [2].

Because the range of serum iPTH and ALP values was large, logarithmic transformation was used for evaluation. Creatinine was determined as the percentage of the median according to the age of Japanese patients $(\% \mathrm{Cr})$ [7]. Serum ALP values were measured by the Japan Society of Clinical Chemistry method.

All data including serum ALP and iPTH were obtained before the therapy for vitamin D-deficient rickets except for the case of the linear mixed model, where we used time-dependent data.

\section{Statistical analysis}

We selected the age, sex, and body mass index (BMI) standard deviation (SD) values as important variables associated with MDA. We also selected serum calcium, phosphorus, log iPTH, log ALP, \%Cr, 25hydroxyvitamin D (25(OH)D), and 1,25-dihydroxyvitamin $\mathrm{D}\left(1,25(\mathrm{OH})_{2} \mathrm{D}\right)$ levels as markers of bone metabolism. The association of MDA with other variables was analyzed by decision tree analysis, Spearman's correlation analysis, and multiple regression analyses (MRA).

Decision tree analysis is an automatic method of determining a variable that has a strong impact on another variable [8]. Since the output is the rule of division, the result is readily understandable. Decision tree analysis involves a roundup analysis of multivariable data to determine the variables and thresholds that have the most impact. It repeatedly divides the segmented targets further, and analysis ends when it is no longer necessary to make a selection.

Then, we analyzed the variables associated with MDA using a linear mixed model, which is suitable for analysis of the longitudinal data of patients [9]. Because the nominal variable could not be analyzed in the linear mixed model by random intercept, sex was excluded from this analysis.

Based on these results, we created a path model [10]. First, we created paths from each variable that showed a significant $(p<0.05)$ correlation in the above analyses of MDA. Next, paths were added between each variable that showed a significant correlation $(p<0.05)$. Finally, error variables were added to each variable. Then, we examined the data by structural equation modeling (SEM) [10] .

Beran et al. [10] reported that this analysis was equal or superior to conventional regression analyses in terms of statistical power. In fact, because of its usefulness, medical science has increasingly adopted it. SEM is an analytical method of testing causal hypotheses for an event. SEM is an extension of factor analysis and multiple linear regression analysis and typically yields a path diagram showing the direction and strength of causal relationships between variables $[10,11]$. Especially, this method enables us to evaluate both direct and total (direct plus indirect) effects. In detail, measured variables were put into the model and various models were tested for goodness of fit, which is usually judged from the lowest RMSEA (root mean square error of approximation) values of $<0.05$, an AIC (Akaike information criterion) score as low as possible, and a CFI (comparative fit index) score as near to 1 as possible. To evaluate the significance in the total effect of path analysis, we used the Bayesian method [12] in AMOS 23.0 to calculate estimated values for the mean and standard error (SE). The Bayesian method allows parameter estimation with small samples by analyzing the mean and variance values of model parameters in the population [12]. We used a coefficient of 3.29 for SE to evaluate a $p$-value of $<0.001$ [13]. In detail, when the mean $+/-$ SE values multiplied by 3.29 did not straddle a value of 0 , then the $p$-value for the item was $<0.001$. Variables with a $p$-value $<0.001$ and absolute value of standardized total effects no less than 0.3 were considered significant factors for 
MDA. To overcome the problem of multiplicity, $p$-values $<0.0241$ were regarded as significant according to the false discovery rate [14].

Finally, neural network analysis (Nnet analysis) [15-17] was performed because it is useful for efficiently predicting several events. This analysis places an intermediate layer between the input and output variables and sets appropriate weights for each variable, which results in more accurate predictions with less prediction error. Neural network analysis can also be used to analyze data with a nonlinear distribution [15]. We examined whether it is possible to predict the presence or absence of genu varum by Nnet analysis. We used parameters that showed significant correlations with MDA by MRA and a path analysis for input variables, and MDA for an output variable. Three-dimensional diagrams by Nnet analysis were used to identify the association between MDA values and $\% \mathrm{Cr}, \log \mathrm{ALP}$, and $\log \mathrm{iPTH}$ values. JMP version 8.0 (SAS Institute, Cary, NC, USA) was used for the decision tree, multiple regression, and Nnet analyses. SPSS version 23.0 (IBM Corp., Armonk, NY, USA) was used for Spearman's correlation analysis and a linear mixed model analysis. AMOS 23.0 (IBM-SPSS, USA) was used for SEM.

\section{Results}

On entry to this study, 42 children were clinically diagnosed with vitamin D deficiency or insufficiency. We excluded 10 children who were too young to walk by themselves and two children with fewer than three visits. Profiles of the 30 selected children are shown in Table 1, where we included information on dietary restrictions due to food allergy and inadequate sunlight exposure. There were no patients with an unbalanced diet due to autistic disorders in our study. The age of patients was $23.3 \pm 8.3$ (mean $\pm \mathrm{SD}$ ) months, comprising of 13 boys and 17 girls. Serum 25(OH)D levels were measured in 25 patients. Pretreatment values of laboratory data are shown in Table 2. All the children had bone lesions of rickets based on roentgenograms. We could not judge the presence of genu varum in two children because they were not examined by roentgenograms for the lower limbs at the first visit. Overall, 16 children had genu varum.

In 28 children, $\% \mathrm{Cr}, \log \mathrm{ALP}$, and $\log \mathrm{iPTH}$ were selected as variables related to MDA by decision tree analysis. BMI SDS was not selected as an important factor associated with MDA (Fig. 1). Also, only the $\% \mathrm{Cr}$ value was selected as a variable associated with MDA by Spearman's correlation analysis $(p=0.026$, Table 3$)$,

Table 1 Patient profile

\begin{tabular}{lccc}
\hline & positive & negative & percentage (\%) \\
\hline dietary restrictions due to food allergies & 25 & 5 & 83 \\
Inadequate sunlight exposure & 8 & 16 & 33 \\
bone lesions & 30 & 0 & 100 \\
genu varum & 16 & 12 & 57 \\
measurement of $25(\mathrm{OH})$ vitamin D & 25 & 5 & 83 \\
vitamin D deficiency & 23 & 2 & 92 \\
vitamin D insufficiency & 25 & 0 & 100 \\
administration of $1 \alpha-\mathrm{OH}-\mathrm{D}_{3}$ & 30 & 0 & 100 \\
administration of calcium preparation & 5 & 25 & 17 \\
\hline
\end{tabular}

Vitamin D deficiency was denoted as serum $25(\mathrm{OH})$ vitamin D levels were no more than $20 \mathrm{ng} / \mathrm{mL}$. Vitamin D insufficiency was denoted as serum 25(OH) vitamin D levels were between 20 and $30 \mathrm{ng} / \mathrm{mL}$.

Table 2 Laboratory data

\begin{tabular}{lccc}
\hline & median & $25-75$ percentile & $n$ \\
\hline \%creatinine $(\%)$ & 83 & $58-87$ & 29 \\
metaphyseal diaphyseal angle (degree) & 12 & $5.8-16$ & 28 \\
intact PTH $(\mathrm{pg} / \mathrm{mL})$ & 180 & $130-320$ & 29 \\
alkaline phosphatase $(\mathrm{IU} / \mathrm{L})$ & 2,200 & $1,500-3,200$ & 28 \\
$25(\mathrm{OH})$ vitamin D $(\mathrm{ng} / \mathrm{mL})$ & 10 & $6.7-14$ & 25 \\
$1,25(\mathrm{OH})_{2}$ vitamin D $(\mathrm{pg} / \mathrm{mL})$ & 130 & $86-190$ & 27 \\
\hline
\end{tabular}

Data were obtained from patients with vitamin D-deficient rickets before the treatment. All laboratory data were obtained from serum samples. 
where we included BMI SDS values. Sex and age were significantly correlated with $\log$ iPTH (Table 4). It revealed that $\log \mathrm{ALP}$ and $\% \mathrm{Cr}$ were significantly correlated with MDA values by multiple regression analysis ( $p=0.012,0.021$, respectively, Table 5). Regarding the linear mixed model, the association between each variable is shown in Table 6 . The significant factor for MDA was $\log \operatorname{ALP}(p=0.021)$.

We created a path model for MDA based on these results. This model was significant with the value of RMSEA $<0.0001$, CFI 1.00, AIC 71.9, and $r^{2} 0.45$ (Fig. 2). Fig. 3 shows the data of the standardized total effect in terms of the mean and SE value for each variable in the path analysis as we used this type of presentation previously [18]. It revealed that the $\log$ iPTH values had a significant effect on increasing MDA values. On the other hand, the log ALP values had a significant effect on reducing MDA values. Although the $\% \mathrm{Cr}$ values were not significant in terms of a path coefficient value, they decreased the MDA values in terms of the $p$-value.

On the basis of these results, age, sex, \%Cr, log ALP, and $\log \mathrm{iPTH}$ were selected as factors directly or indirectly related to MDA values. Then, we performed Nnet analysis using them as input variables and MDA values as output variables (Fig. 4). The $r^{2}$ value between measured and predicted MDA values was 0.93 , while the cross validation (5-fold) value of $r^{2}$ was 0.51 . For predicting genu varum, it revealed a sensitivity of $79 \%$, specificity of $100 \%$, and c-index of 0.89 . A threedimensional diagram revealed that MDA values decreased as standardized log ALP values increased when standardized log iPTH values were elevated above about 0.6. In contrast, MDA values increased as standardized log ALP values increased when standardized log iPTH values were elevated to about 0.4 or lower, and MDA values tended to increase as standardized log iPTH values increased (Fig. 5). As \% $\mathrm{Cr}$ values increased, MDA values decreased (Fig. 6).

\section{Discussion}

A biphasic association of serum ALP levels with MDA was shown to be dependent on the stage of rickets judging from serum iPTH levels [5]. The serum ALP level is considered to reflect the presence of rickets [6], while it is also regarded as a bone formation marker in the field of osteoporosis [19]. Recently, MDA has been used as a quantitative index of genu varum [2]; however, there are no reports on the association of serum ALP levels with MDA in children with vitamin D-deficient rickets. In this study, we clarified for the first time the clinical significance of serum ALP levels in patients with

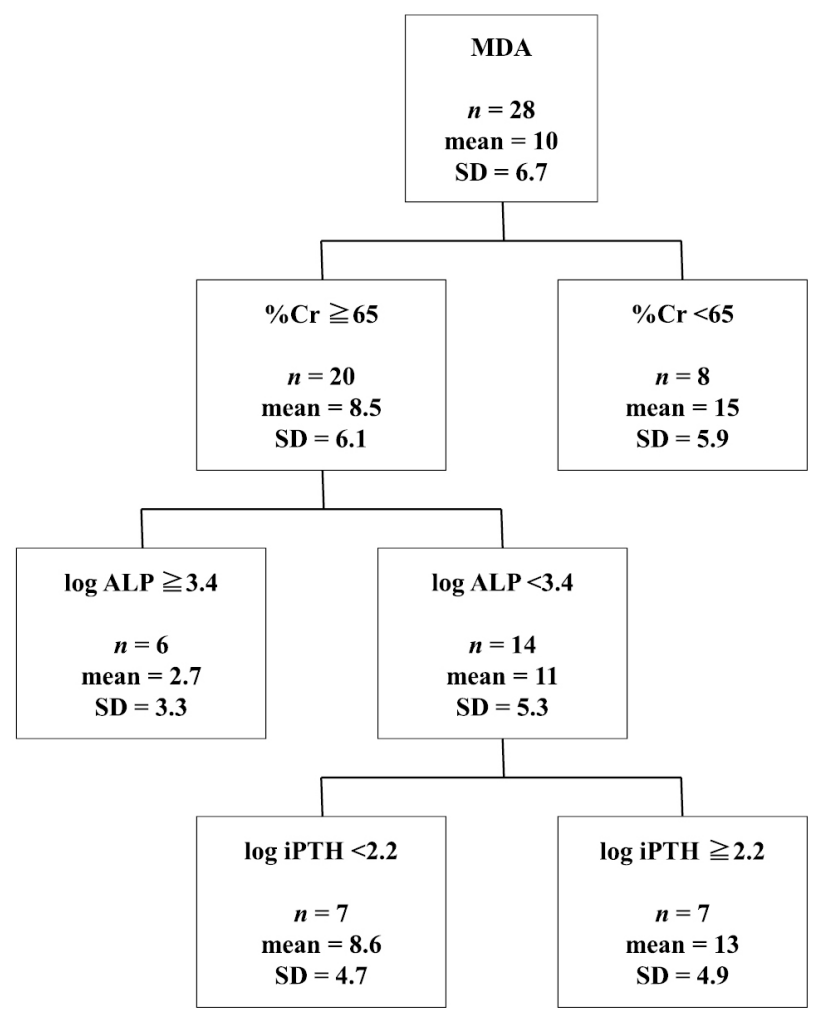

Fig. 1 Decision tree analysis.

MDA, \%Cr, $\log$ ALP, and log iPTH denoted metaphyseal diaphyseal angle, \%Creatinine, log alkaline phosphatase, and $\log$ intact PTH, respectively. We entered all data listed in Table 3 in the analysis. Then, \%Creatinine, log alkaline phosphatase, and log intact PTH were selected as variables associated with MDA, in descending order of relevance. $r^{2}$ value: $0.52, \quad r^{2}$ value (5-fold crossvalidation): 0.42 .

genu varum due to vitamin D-deficient rickets.

In detail, we revealed that an increase in the serum ALP level was associated with deteriorating genu varum when the serum iPTH level was slightly elevated, which is considered to be an early stage of vitamin D-deficient rickets [5]. Although we assumed a causal association between ALP and genu varum because of the retrospective nature of the study, these findings were considered to be reasonable because a decreased bone quality due to rickets [20] would lead to a decrease in bone strength of the lower extremities. This is because bone quality can explain $30 \%$ of the bone strength [21]. On the other hand, we found greater increases of serum ALP levels in advanced stages of rickets based on elevated serum iPTH levels, focused on as an associated factor to ameliorate genu varum. This was an unexpected observation because it could not be explained by the mechanism of decreased bone quality due to rickets.

In fact, Sakamoto et al. [3] reported weak but significant positive correlations of serum ALP levels with 
Table 3 Factors associated with the metaphyseal diaphyseal angle by Spearman's correlation analyses

\begin{tabular}{lcc}
\hline variable & correlation coefficient & $p$-value \\
\hline age (month) & -0.011 & 0.96 \\
sex (male for 1) & -0.15 & 0.44 \\
body mass index (SDS) & -0.045 & 0.82 \\
age at the first independent walk (month) & 0.37 & 0.14 \\
dietary restrictions (positive for 1) & -0.098 & 0.62 \\
\hline sunlight exposure (lack for 1) & 0.27 & 0.22 \\
calcium (mg/dL) & 0.033 & 0.87 \\
phosphorus (mg/dL) & 0.084 & 0.67 \\
\%creatinine (\%) & $-\mathbf{0 . 4 2}$ & $\mathbf{0 . 0 2 6}$ \\
\hline $\log$ intact PTH (pg/mL) & 0.32 & 0.11 \\
log alkaline phosphatase (IU/L) & -0.26 & 0.20 \\
\hline $25(\mathrm{OH})$ vitamin D $(\mathrm{ng} / \mathrm{mL})$ & -0.22 & 0.32 \\
$1,25(\mathrm{OH})_{2}$ vitamin D $(\mathrm{pg} / \mathrm{mL})$ & -0.099 & 0.64 \\
\hline
\end{tabular}

Bold letters denoted $p$-values less than 0.05 . All laboratory data were obtained from serum samples.

Table 4 Associations between each variable by Spearman's correlation analyses

\begin{tabular}{|c|c|c|c|c|c|c|c|}
\hline & age & $\operatorname{sex}$ & $\% \mathrm{Cr}$ & $\log \mathrm{iPTH}$ & $\log$ ALP & $25(\mathrm{OH})$ vitamin D & $1,25(\mathrm{OH})_{2}$ vitamin $\mathrm{D}$ \\
\hline age (month) & 1.0 & $\begin{array}{c}-0.090 \\
(0.64)\end{array}$ & $\begin{array}{c}-0.071 \\
(0.72)\end{array}$ & $\begin{array}{c}0.41 \\
(0.026)\end{array}$ & $\begin{array}{l}0.080 \\
(0.68)\end{array}$ & $\begin{array}{c}-0.092 \\
(0.66)\end{array}$ & $\begin{array}{c}-0.049 \\
(0.81)\end{array}$ \\
\hline sex (male for 1 ) & & 1.0 & $\begin{array}{c}0.21 \\
(0.28)\end{array}$ & $\begin{array}{c}-0.50 \\
(0.005)\end{array}$ & $\begin{array}{l}0.082 \\
(0.68)\end{array}$ & $\begin{array}{c}-0.022 \\
(0.92)\end{array}$ & $\begin{array}{c}0.16 \\
(0.44)\end{array}$ \\
\hline$\% \mathrm{Cr}(\%)$ & & & 1.0 & $\begin{array}{l}-0.30 \\
(0.13)\end{array}$ & $\begin{array}{l}-0.19 \\
(0.35)\end{array}$ & $\begin{array}{c}0.17 \\
(0.42)\end{array}$ & $\begin{array}{c}0.17 \\
(0.41)\end{array}$ \\
\hline $\log$ iPTH $(\mathrm{pg} / \mathrm{mL})$ & & & & 1.0 & $\begin{array}{c}0.27 \\
(0.16)\end{array}$ & $\begin{array}{l}-0.22 \\
(0.30)\end{array}$ & $\begin{array}{l}-0.16 \\
(0.44)\end{array}$ \\
\hline $\log \operatorname{ALP}(\mathrm{IU} / \mathrm{L})$ & & & & & 1.0 & $\begin{array}{c}0.14 \\
(0.51)\end{array}$ & $\begin{array}{c}-0.14 \\
(0.50)\end{array}$ \\
\hline $25(\mathrm{OH})$ vitamin $\mathrm{D}(\mathrm{ng} / \mathrm{mL})$ & & & & & & 1.0 & $\begin{array}{c}0.31 \\
(0.15)\end{array}$ \\
\hline $1,25(\mathrm{OH})_{2}$ vitamin $\mathrm{D}(\mathrm{pg} / \mathrm{mL})$ & & & & & & & 1.0 \\
\hline
\end{tabular}

Figures in Table 4 were correlation coefficients. Data in the parentheses were $p$-values. Cr, iPTH, and ALP denoted creatinine, intact PTH, and alkaline phosphatase, respectively. Bold letters denoted $p$-values less than 0.05 .

Table 5 Associations between the metaphyseal diaphyseal angle and each variable by the multiple regression analysis

\begin{tabular}{lcc}
\hline Parameters & standardized $\beta$ value & $p$-value \\
\hline \%creatinine (\%) & $\mathbf{- 0 . 4 8}$ & $\mathbf{0 . 0 2 1}$ \\
$\log$ intact PTH $(\mathrm{pg} / \mathrm{mL})$ & 0.074 & 0.71 \\
$\log$ alkaline phosphatase (IU/L) & $\mathbf{- 0 . 4 7}$ & $\mathbf{0 . 0 1 2}$ \\
\hline
\end{tabular}

A $p$-value by analysis of variance was 0.010 . Values for $\mathrm{R}^{2}$ and corrected $\mathrm{R}^{2}$ were 0.40 and 0.31 , respectively. Bold letters denoted $p$-values less than 0.05 .
Table 6 Associations between the metaphyseal diaphyseal angle and each variable by the linear mixed model

\begin{tabular}{lcc}
\hline Parameters & Estimate & $p$-value \\
\hline age (month) & -0.31 & 0.25 \\
\hline \%creatinine (\%) & $\underline{-0.52}$ & $\underline{0.052}$ \\
\hline $25(\mathrm{OH})$ vitamin D $(\mathrm{ng} / \mathrm{mL})$ & 0.14 & 0.82 \\
$1,25(\mathrm{OH})_{2}$ vitamin D $(\mathrm{pg} / \mathrm{mL})$ & -0.019 & 0.67 \\
$\log$ intact PTH $(\mathrm{pg} / \mathrm{mL})$ & 18 & 0.17 \\
log alkaline phosphatase $(\mathbf{I U} / \mathbf{L})$ & $\mathbf{- 4 5}$ & $\mathbf{0 . 0 2 1}$ \\
\hline
\end{tabular}

Bold letters denoted $p$-values less than 0.05 . Underlined letters denoted $p$-values between 0.05 and 0.06 . 
MDA values in children with physiological genu varum. However, there were several differences between the previous study and ours. For example, they only showed cross-sectional data for analysis. Also, there are theoretical differences between physiological genu varum and vitamin D-deficient rickets. Moreover, they did not include the serum creatinine level, considered to be a marker of muscle mass [4]. Recently, the muscle bone axis has become a topic in bone metabolism [22]. In fact, decreased muscle mass such as sarcopenia was

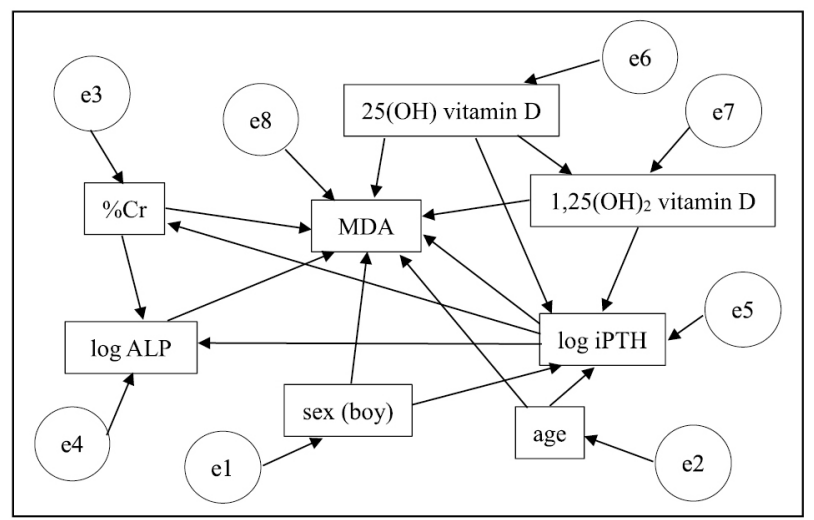

Fig. 2 Path model for MDA.

Paths from sex and age to $\log$ iPTH were set according to the results of monovariate analyses. Paths from $25(\mathrm{OH})$ vitamin $\mathrm{D}$ to $1,25(\mathrm{OH})_{2}$ vitamin $\mathrm{D}$ were set according to the metabolic path of vitamin D. e denoted error. We analyzed this path diagram using structural equation modeling, which allows us to test the model hypothesis. The path was significant with RMSEA $<0.0001$, CFI $=$ $1.00, \mathrm{AIC}=72, r^{2}=0.45$. reported to be related to bone loss in adult patients [23]. Also, we noted a positive association of the $\% \mathrm{Cr}$ value, a marker of muscle mass, to improve genu varum in this study. Thus, the presence or absence of serum creatinine levels in analyses may lead to different results.

The serum ALP level is also a marker of bone formation [19]. Thus, we considered the elevation of the serum ALP value in the advanced stage of rickets to be due to increased bone formation caused by mechanical stress [24] with the progression of genu varum.

We did not find any reports on humans describing the detailed mechanism leading to the association of mechanical stress with ALP activity and bone formation. However, we identified a study investigating these issues using human bone samples and ex-vivo experiments [25]. It showed increases in ALP activity and the bone formation rate in bone specimens caused by cyclical mechanical stimulation, which simulates daily jumping exercise for 5 minutes. The researchers indicated that decreased osteocyte apoptosis was involved in the mechanism. This is an interesting report since osteocytes are considered to work as mechano-sensors in bones. For the in vitro data, some reports suggested increased or decreased ALP activity of osteoblasts in culture by mechanical stress or zero gravity, respectively [26, 27]. All these data support our hypothesis that mechanical stress is involved in improving genu varum due to vitamin D-deficient rickets through increased bone formation by increased tortion mechanical stress in the lower extremities. Further study is necessary to elucidate this.

The second finding was the fact that the serum iPTH level was associated with deteriorating genu varum based on

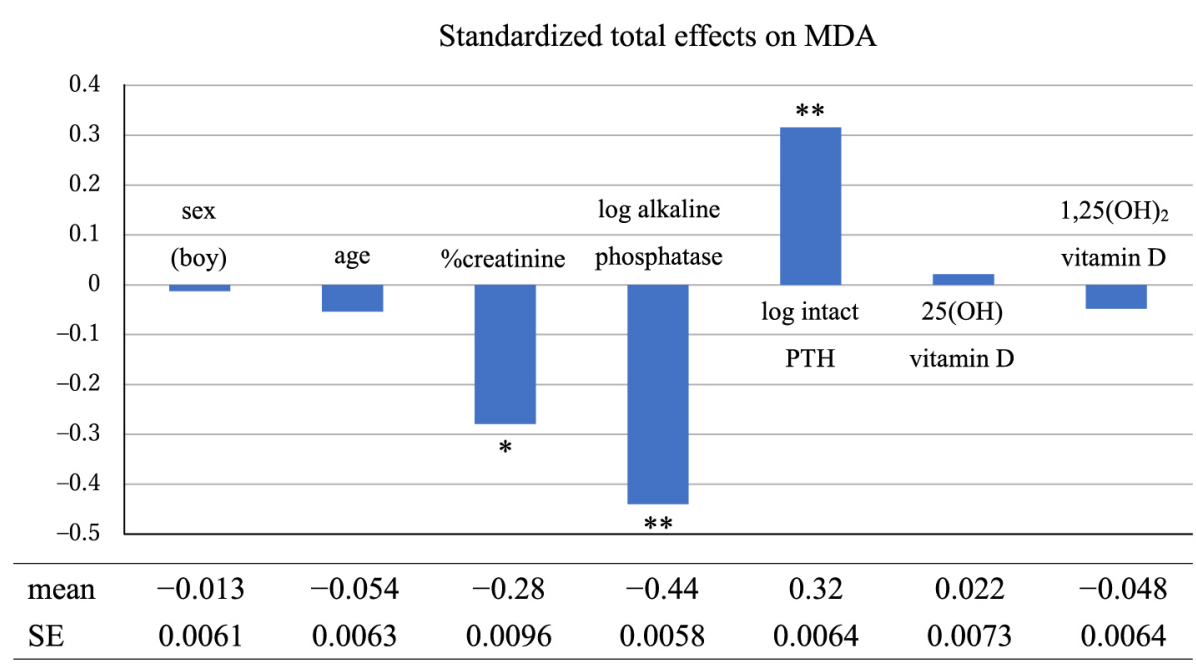

Fig. 3 Standardized total effect of each variable on MDA.

The path model in Fig. 2 was tested by structural equation modeling and the model was significant. The standardized total effect that actually represents the influence of each variable on MDA is shown. Significant variables were denoted as (**), when they had a standardized total effect no less than 0.3 with a $p$-value $<0.001$. Variables denoted $\left({ }^{*}\right)$ indicated the total effects from 0.2 to 0.3 with $p$-values $<0.001$. SE denoted standardized error. 


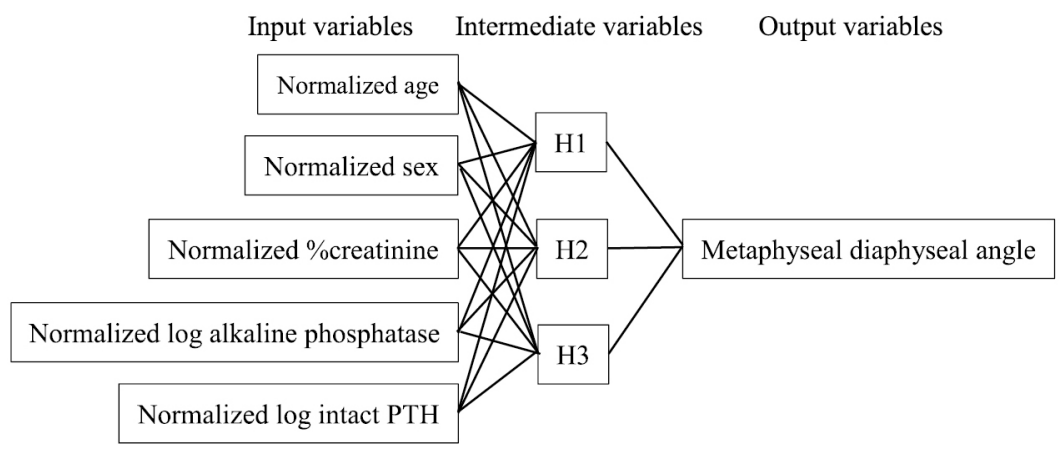

Fig. 4 Neural network to predict the presence or absence of genu varum.

The model of neural network analysis is presented. The analysis was performed with age, sex, \%creatinine, log alkaline phosphatase, and log intact PTH as input variables and the metaphyseal diaphyseal angle (MDA) as an output variable. All values except MDA were standardized. Three nodes (H1, H2, and H3) were set in the intermediate layer, which enabled us to conduct more accurate predictions with less prediction error.

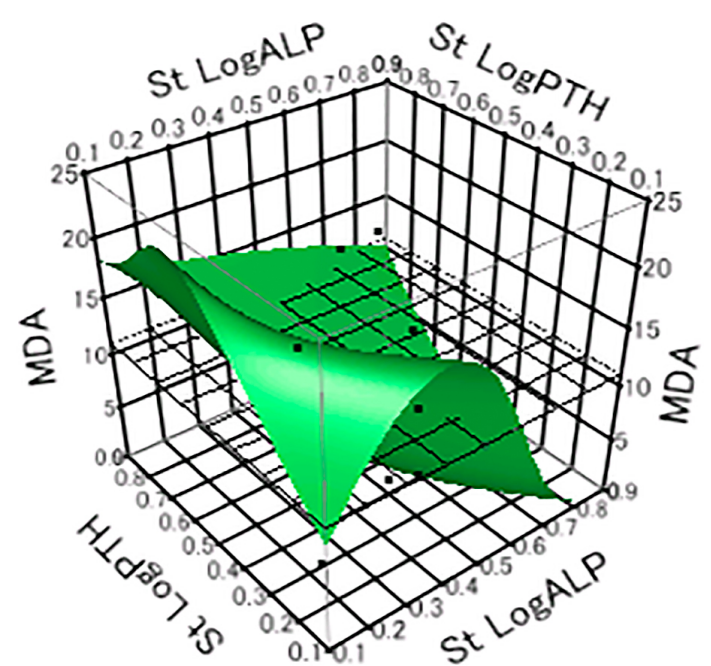

Fig. 5 Three-dimensional diagram indicating the associations among log intact PTH, log alkaline phosphatase, and the metaphyseal diaphyseal angle.

St $\operatorname{LogPTH}$, St LogALP, and MDA denote standardized $\log$ intact PTH, standardized log alkaline phosphatase, and the metaphyseal diaphyseal angle, respectively. A meshed plane indicates MDA values with 11 degrees, which was a criterion to differentiate genu varum from normal values. MDA values decreased as St $\log$ ALP values increased when $\mathrm{St} \log \mathrm{PTH}$ values were above about 0.6. At lower St $\log \mathrm{PTH}$ values (about $0.1-0.4$ ), MDA increased as St $\log$ ALP increased to about 0.4. However, when St $\log$ ALP was above 0.4, MDA decreased as St $\log$ ALP increased further.

path and Nnet analyses. Since iPTH was reported to be related to genu valgum in hyperparathyroidism, [28, 29] this might be related to the deterioration of genu varum through reducing the bone strength in the lower extremities.

Log iPTH was selected as a variable related to MDA by decision tree analysis along with $\% \mathrm{Cr}$ and $\log$ ALP (Fig. 1). Both \%Cr and $\log$ ALP have been shown to be

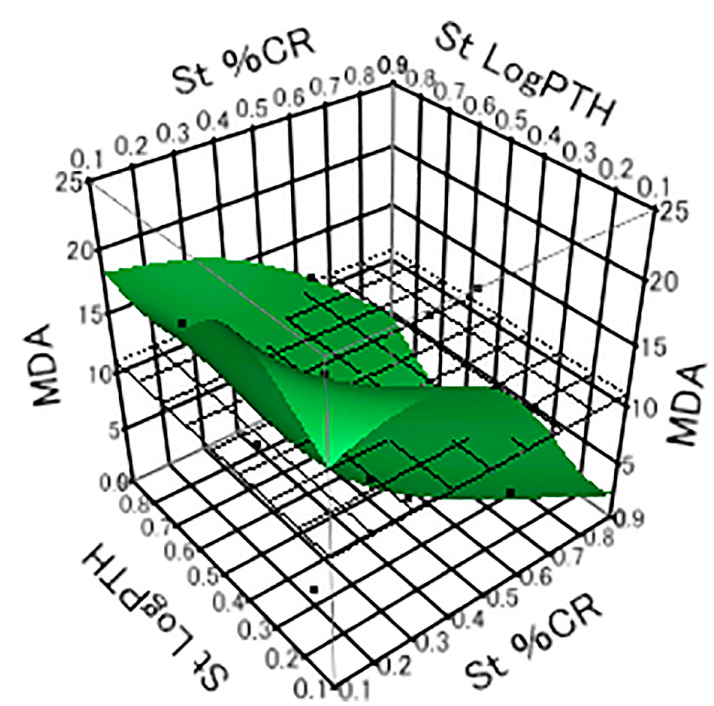

Fig. 6 Three-dimensional diagram indicating the associations among log intact PTH, \%creatinine, and the metaphyseal diaphyseal angle.

St LogPTH, St \%CR, and MDA denote standardized $\log$ intact PTH, standardized \%creatinine, and the metaphyseal diaphyseal angle, respectively. We used a meshed plane as indicated in Fig. 5. As St \%CR increased, MDA tended to decrease.

related to MDA also by other analytical methods, but there were no significant relationships between log iPTH and MDA revealed by Spearman's correlation analysis (Table 3), multiple regression analysis (Table 5) or linear mixed model (Table 6). The latter three analyses were suitable for analyzing the data with linear associations. By contrast, the decision tree analysis was reported to be useful for analyzing both linear and non-linear data [30]. Thus, this difference was speculated to be the reason why the $\log$ iPTH was shown to be a variable associated with MDA by only the decision tree analysis. 
We used MRA, path, and linear mixed model analyses. These analyses were set to deal with linear distributions of measured data. In addition, the linear mixed model made it possible to analyze time-dependent data, which usually indicate more plausible causal relationships in clinical studies by reducing measurement errors [31]. Thus, the positively associated data of serum ALP with improving MDA values in vitamin D-deficient rickets in this study are considered to reflect the actual situation.

Moreover, we used Nnet analysis, which can analyze data with a non-linear distribution based on the logic similar to the human neuron network [15]. In our study, we noted a biphasic association of serum ALP levels with MDA values: an initial deteriorative association in contrast to an ameliorative association in the advanced stage of rickets judging from serum iPTH levels [5]. This is considered to be derived from the fact that the small change of deteriorative associations could only be detected by Nnet analysis since it was regarded as trivial by analyses dependent on the data with linear distributions. Further study is necessary to clarify this.

Several limitations of this study are acknowledged. First, the number of cases with vitamin D-deficient rickets was small compared with that in previous studies employing path [32] and Nnet [33] analyses. However, the former analysis in our study was highly significant with RMSEA $<0.0001$ and $p<0.0001$. Also, the latter analysis had a useful clinical meaning because of $r^{2}$ values of $0.93(r=0.96)$ and $0.51(r=0.71)$ by the 5 -fold cross-validation method. In addition, sensitivity and specificity to predict genu varum was 79 and $100 \%$ respectively. All these data suggest the suitability of our findings to generate a working hypothesis.

Second, we did not investigate the presence of calcium deficiency in this study, which is known to occur in patients with vitamin D-deficient rickets [34]. Therefore, the effects of calcium deficiency on genu varum in asso- ciation with vitamin D deficiency should be investigated in the future.

Third, we did not measure serum zinc levels in this study. Since they were reported to affect serum ALP levels [35], this might affect our results of an association of serum ALP levels with genu varum. However, we could exclude this possibility because we used linear mixed models to analyze time-dependent data including ALP, which enabled us to exclude the effect of individual differences of serum zinc levels due to the different lifestyle of pediatric patients in our study. Further study is necessary to elucidate this.

Fourth, we did not measure bone mineral densities or muscle masses in the lower extremities. This will be necessary in future studies.

In conclusion, we identified a biphasic association of serum ALP levels with the severity of genu varum in pediatric patients with vitamin D-deficient rickets. We revealed that serum ALP levels had an ameliorative association with genu varum. This is the first study to reveal that bone formation due to torsion mechanical stress in humans might contribute to the improvement of genu varum through the elevation of serum ALP levels. These findings are important and informative for pediatricians to understand the meanings of serum ALP levels in genu varum on treating patients with vitamin D-deficient rickets.

\section{Acknowledgement}

The authors thank all participating children, parents, and all medical staff at the participating institutions for providing clinical data.

\section{Disclosure}

The authors declare no competing interests.

\section{References}

1. Rosenfeld SB (2020) Approach to the child with bowlegs. In: UpToDate, Phillips WA, Drutz JE (Ed), Retrieved August 19, 2020. from https://www.uptodate.com/ contents/approach-to-the-child-with-bow-legs accessed on September 2020

2. Levine AM, Drennan JC (1982) Physiological bowing and tibia vara. The metaphyseal-diaphyseal angle in the measurement of bowleg deformities. J Bone Joint Surg Am 64: 1158-1163.

3. Sakamoto Y, Ishijima M, Kinoshita M, Liu L, Suzuki M, et al. (2018) Association between leg bowing and serum alkaline phosphatase level regardless of the presence of a radiographic growth plate abnormality in pediatric patients with genu varum. J Bone Mineral Metab 36: 447-453.

4. Schutte JE, Longhurst JC, Gaffney FA, Bastian BC, Blomqvist CG (1981) Total plasma creatinine: an accurate measure of total striated muscle mass. J Appl Physiol Respir Environ Exerc Physiol 51: 762-766.

5. Levine M, Zapalowski C, Kappy M (2005) Disorders of calcium, phosphate, PTH and vitamin D metabolism. In: Kappy MS, Allen DB, Geffner ME (ed), Principles and Practice of Pediatric Endocrinology. Charles C. Thomas Publisher, Springfield, USA: 762.

6. Fukumoto S, Ozono K, Michigami T, Minagawa M, Okazaki R, et al. (2015) Pathogenesis and diagnostic criteria for rickets and osteomalacia-Proposal by an expert 
panel supported by Ministry of Health, Labour and Welfare, Japan, The Japanese Society for Bone and Mineral Research and The Japan Endocrine Society. Endocr J 62: 665-671.

7. Uemura O, Honda M, Matsuyama T, Ishikura K, Hataya H (2011) Age, sex, and body length effects on reference serum creatinine levels determined by an enzymatic method in Japanese children: a multicenter study. Clin Exp Nephrol 15: 694-699.

8. Podgorelec V, Kokol P, Stiglic B, Rozman I (2002) Decision trees: an overview and their use in medicine. $\mathrm{J} \mathrm{Med}$ Syst 26: 445-463.

9. Casals M, Farrés MG, Carrasco JL (2014) Methodological quality and reporting of generalized linear mixed models in clinical medicine (2000-2012): a systematic review. PLoS One 9: e112653.

10. Beran TN, Violato C (2010) Structural equation models in medical research: a primer. BMC Res Notes 3: 267.

11. Bentler PM, Stein JA (2010) Structural equation models in medical research. Stat Methods Med Res 1: 159-181.

12. Kaplan D, Depaoli S (2012) Bayesian structural equation modeling. In: Hoyle RH (ed), Handbook of structural equation modeling. The Guilford Press, New York, USA: 650-673.

13. Yamamoto T, Mizoguchi Y, Kaneno H, Yamamoto K, Inoue $Y$, et al. (2013) Serum immunoglobulin G subclass levels and estimated clinical severity caused by possible influenza A (H1N1) pdm 2009 infection. J Infect Chemother 19: 833-842.

14. Benjamini Y, Hochberg Y (1995) Controlling the false discovery rate: a practical and powerful approach to multiple testing. J R Stat Soc Series B Stat Methodol 57: 289 300 .

15. Specht DF (1991) A general regression neural network. IEEE Trans Neural Netw 2: 568-576.

16. Shioji M, Yamamoto $\mathrm{T}$, Ibata $\mathrm{T}$, Tsuda $\mathrm{T}$, Adachi $\mathrm{K}$, et al. (2017) Artificial neural networks to predict future bone mineral density and bone loss rate in Japanese postmenopausal women. BMC Res Notes 10: 590.

17. Azuma J, Yamamoto T, Nitta M, Hasegawa Y, Kijima E, et al. (2020) Structure equation model and neural network analyses to predict coronary artery lesions in Kawasaki disease: a single-centre retrospective study. Sci Rep 10: 11868.

18. Yamamoto $\mathrm{T}$, Ihashi $\mathrm{M}$, Mizoguchi $\mathrm{Y}$, Kaneno $\mathrm{H}$, Yamamoto K, et al. (2013) Early therapy with neuraminidase inhibitors for influenza A (H1N1) pdm 2009 infection. Pediatr Int 55: 714-721.

19. Karunaratne A, Esapa CR, Hiller J, Boyde A, Head R, et al. (2012) Significant deterioration in nanomechanical quality occurs through incomplete extrafibrillar mineralization in rachitic bone: evidence from in-situ synchrotron X-ray scattering and backscattered electron imaging. $J$ Bone Miner Res 27: 876-890.

20. Lumachi F, Ermani M, Camozzi V, Tombolan V, Luisetto G (2009) Changes of bone formation markers osteocalcin and bone-specific alkaline phosphatase in postmenopausal women with osteoporosis. Ann N Y Acad Sci 1173 Suppl 1: E60-E63.

21. NIH Consensus Development Panel on Osteoporosis Prevention, Diagnosis, and Therapy (2001) Osteoporosis, prevention, diagnosis, and therapy. JAMA 285: 785-795.

22. Kaji H (2014) Interaction between muscle and bone. $J$ Bone Metab 21: 29-40.

23. Hart $\mathrm{NH}$, Nimphius $\mathrm{S}$, Rantalainen $\mathrm{T}$, Ireland $\mathrm{A}$, Siafarikas A, et al. (2017) Mechanical basis of bone strength: influence of bone material, bone structure and muscle action. J Musculoskelet Neuronal Interact 17: 114-139.

24. Turner CH (2008) Skeletal adaptation to mechanical loading. Clin Rev Bone Miner Metab 5: 181-194.

25. Mann V, Huber C, Kogianni G, Jones D, Noble B (2006) The influence of mechanical stimulation on osteocyte apoptosis and bone viability in human trabecular bone. $J$ Musculoskelet Neuronal Interact 6: 408-417.

26. Nishioka S, Fukuda K, Tanaka S (1993) Cyclic stretch increases alkaline phosphatase activity of osteoblast-like cells: a role for prostaglandin $\mathrm{E}_{2}$. Bone Miner 21: 141150.

27. Carmeliet G, Nys G, Bouillon R (2009) Microgravity reduces the differentiation of human osteoblastic MG-63 cells. J Bone Miner Res 12: 786-794.

28. Sharma S, Kumar S (2016) Bilateral genu valgum: an unusual presentation of juvenile primary hyperparathyroidism. Oxf Med Case Reports 2016: 141-143.

29. Ratnasingam J, Tan ATB, Vethakkan SR, Paramasivam SS, Ibrahim L, et al. (2013) Primary hyperparathyroidism: a rare cause of genu valgus in adolescence. J Clin Endocrinol Metab 98: 869-870.

30. Friedl MA, Brodley CE (1997) Decision tree classification of land cover from remotely sensed data. Remote Sens Environ 61: 399-409.

31. Wang N, Lin X, Gutierrez RG, Carroll RJ (1998) Bias analysis and SIMEX approach in generalized linear mixed measurement error models. J Am Stat Assoc 93: 249-261.

32. Wolf EJ, Harrington KM, Clark SL, Miller MW (2013) Sample size requirements for structural equation models: an evaluation of power, bias, and solution propriety. Educ Psychol Meas 76: 913-934.

33. Raudys Sj, Jain AK (1991) Small sample size effects in statistical pattern recognition: recommendations for practitioners. IEEE Trans Pattern Anal Mach Intel 13: 252-264.

34. Voloc A, Esterle L, Nguyen TM, Walrant-Debray O, Colofitchi A, et al. (2010) High prevalence of genu varum/valgum in European children with low vitamin D status and insufficient dairy products/calcium intakes. Eur J Endocrinol 163: 811-817.

35. Ray CS, Singh B, Jena I, Behera S, Ray S (2017) Low Alkaline Phosphatase (ALP) in adult population an indicator of Zinc (Zn) and Magnesium (Mg) deficiency. Curr Res Nutr Food Sci 5: 347-352. 